Canadian

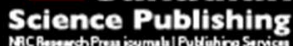

Applied Physiology, Nutrition, and Metabolism Physiologie appliquée, nutrition et métabolisme

\title{
The impact of work-matched interval training on V02peak and V02 kinetics: diminishing returns with increasing intensity
}

\begin{tabular}{|r|l|}
\hline Journal: & Applied Physiology, Nutrition, and Metabolism \\
\hline Manuscript ID & apnm-2015-0614.R1 \\
\hline Manuscript Type: & Article \\
\hline Date Submitted by the Author: & 01-Feb-2016 \\
\hline Complete List of Authors: & $\begin{array}{l}\text { Raleigh, James; Queen's University, Kinesiology } \\
\text { Giles, Matthew; Queen's University, Kinesiology } \\
\text { Scribbans, Trisha; Queen's University, School of Kinesiology and Health } \\
\text { Studies } \\
\text { Edgett, Brittany; Queen's University, School of Kinesiology and Health } \\
\text { Studies } \\
\text { Sawula, Laura; Queen's University, School of Kinesiology and Health } \\
\text { Studies } \\
\text { Bonafiglia, Jacob; Queen's University, School of Kinesiology and Health } \\
\text { Studies } \\
\text { Graham, Ryan; Nipissing University, School of Physical and Health } \\
\text { Education } \\
\text { Gurd, Brendon J.; Queens University, }\end{array}$ \\
\hline Keyword: & \begin{tabular}{l} 
HIIT, VO2peak, VO2 kinetics, exercise training < exercise, non-responders \\
\hline \multirow{2}{*}{ Kend }
\end{tabular} \\
\hline
\end{tabular}

\section{SCHOLARONE}

Manuscripts 
The impact of work-matched interval training on $\mathrm{VO}_{2}$ peak and $\mathrm{VO}_{2}$ kinetics: diminishing returns with increasing intensity

\footnotetext{
James P. Raleigh ${ }^{1}$, Matthew D. Giles ${ }^{1}$, Trisha D. Scribbans ${ }^{1}$, Brittany A. Edgett ${ }^{1}$, Laura J. Sawula $^{1}$, Jacob T. Bonafiglia ${ }^{1}$, Ryan B. Graham ${ }^{2}$ and Brendon J. Gurd ${ }^{1}$

${ }^{1}$ School of Kinesiology and Health Studies, Queen's University, Kingston, Ontario, K7L 3N6, Canada

${ }^{2}$ School of Physical and Health Education, Nipissing University, North Bay, Ontario, P1B 8L7, Canada
}

Corresponding Author:

Brendon J. Gurd, PhD

School of Kinesiology and Health Studies

Queen's University

Kingston, Ontario, K7L 3N6

Telephone: 613-533-6000 ext.79023

Fax: 613-533-2009

Email: gurdb@queensu.ca 


\section{$\underline{\text { Abstract }}$}

High intensity interval training (HIIT) improves $\mathrm{VO}_{2}$ peak and $\mathrm{VO}_{2}$ kinetics, however, it is unknown whether an optimal intensity of HIIT exists for eliciting improvements in these measures of whole body oxidative metabolism. The purpose of this study was to: 1) investigate the effect of interval intensity on training-induced adaptations in $\mathrm{VO}_{2}$ peak and $\mathrm{VO}_{2}$ kinetics, and 2) examine the impact of interval intensity on the frequency of nonresponders in $\mathrm{VO}_{2}$ peak. 36 healthy men and women completed 3 weeks of cycle ergometer HIIT, consisting of intervals targeting $80 \%$ (LO), $115 \%$ (MID) or $150 \%$ (HI) of peak aerobic power. Total work performed per training session was matched across groups. A main effect of training $(p<0.05)$, and a significant interaction effect was observed for $\mathrm{VO}_{2}$ peak, with the change in $\mathrm{VO}_{2}$ peak being greater $(p<0.05)$ in the MID group than the LO group; however, no differences were observed between the HI group and either the MID or LO groups $\left(\Delta \mathrm{VO}_{2}\right.$ peak; LO, $2.7 \pm 0.7 \mathrm{ml} / \mathrm{kg} / \mathrm{min} ; \mathrm{MID}, 5.8 \pm 0.7$; HI, $4.2 \pm 1.0$ ). The greatest proportion of responders was observed in the MID group (LO, 8/12; MID, 12/13; HI, 9/11). A non-significant relationship $\left(p=0.26 ; \mathrm{r}^{2}=0.04\right)$ was found between the changes in $\mathrm{VO}_{2}$ peak and $\tau \mathrm{VO}_{2}$. These results suggest that training at intensities around $\mathrm{VO}_{2}$ peak may represent a threshold intensity above which further increases in training intensity provide no additional adaptive benefit. The dissociation between changes in $\mathrm{VO}_{2}$ peak and $\mathrm{VO}_{2}$ kinetics also reflects the different underlying mechanisms regulating these adaptations.

\section{Key Words: HIIT, $\mathrm{VO}_{2}$ peak, $\mathrm{VO}_{2}$ kinetics, exercise training, non-responders, intensity, work-matched}




\section{Introduction}

High-intensity interval training (HIIT) induces similar improvements in aerobic exercise capacity as moderate-intensity endurance training despite requiring significantly less exercise time (Eddy et al. 1977, Burgomaster et al. 2005, Cocks et al. 2013, Scribbans et al. 2014). While many interval training protocols improve cardiovascular fitness (Gillen and Gibala 2014), training studies comparing different intensities of HIIT have only compared two intensities, have not examine supramaximal intensities, and/or have not compared protocols that were work-matched (Burke et al. 1994, Boyd et al. 2013). Thus, the impact of training intensity for work-matched (i.e. equivalent training session energy expenditure) interval training on improvements in aerobic exercise capacity remains unclear. Further, as a result of this gap in the literature, whether there is an optimal intensity of HIIT for eliciting improvements in aerobic exercise capacity remains unknown.

Peak oxygen uptake ( $\mathrm{VO}_{2}$ peak), an estimate of the maximal ability of the cardiovascular and muscular systems to deliver and utilize oxygen during exercise $\left(\mathrm{O}_{2}\right)$ (Mitchell et al. 1958), is the most commonly utilized indicator of improvements in aerobic exercise capacity in exercise training literature. $\mathrm{VO}_{2}$ kinetics, an alternative measure of whole body oxidative metabolism during exercise, provides information regarding exercising muscle's ability to respond to an instantaneous increase in $\mathrm{O}_{2}$ demand during the transition from rest to exercise (Barstow et al. 1990, Grassi et al. 1996). Both moderate intensity continuous (endurance) training (Carter et al. 2000) and HIIT (Krustrup et al. 2004, Berger et al. 2006, McKay et al. 2009) improve $\mathrm{VO}_{2}$ peak and $\mathrm{VO}_{2}$ kinetics; however, the strongest determinant of $\mathrm{VO}_{2}$ peak in healthy individuals is 
cardiovascular capacity of $\mathrm{O}_{2}$ delivery (Richardson et al. 1993), with $\mathrm{VO}_{2}$ kinetics being more dependent on processes at the site of the muscle (Grassi 2001). There is evidence that adaptations to these two variables can dissociate (Wilkerson et al. 2011, Poole and Jones 2012). Our lab has found a positive effect of intensity on $\mathrm{VO}_{2}$ peak with training with no such effect on mitochondrial content (Boyd et al. 2013), as well as no difference between submaximal and supramaximal training groups on oxidative capacity and capillary density (Scribbans et al. 2014), suggesting that $\mathrm{VO}_{2}$ peak changes in response to training are related to intensity of exercise and that $\mathrm{VO}_{2}$ kinetics changes are not. Thus, it is logical to posit that changes in $\mathrm{VO}_{2}$ peak and $\mathrm{VO}_{2}$ kinetics would not be uniform in response to different intensities of HIIT. It also remains unclear whether an optimal intensity of HIIT exists for inducing improvement in $\mathrm{VO}_{2}$ peak and/or $\mathrm{VO}_{2}$ kinetics.

In addition to establishing the impact of exercise intensity on group training responses, the importance of examining individual responses to exercise training is becoming increasingly evident given recent demonstrations of inter-individual heterogeneity and the existence of individuals who fail to adapt to training (i.e. nonresponders) (Bouchard and Rankinen 2001, Hautala et al. 2006, Sisson et al. 2009). Genetics, age, sex and baseline cardiovascular function all influence an individual's response to exercise training (Cunningham et al. 1987, Bouchard et al. 1999, Hautala et al. 2003, Sisson et al. 2009). There is also evidence that increases in training dose (i.e. weekly $\mathrm{kcal} / \mathrm{kg}$ expenditure) contribute to a greater magnitude of training response (Sisson et al. 2009), suggesting that any increases in exercise stimulus (intensity, volume, etc.) may result in more widespread responses in individuals with low sensitivity to exercise training (Buford et al. 2013). While it has been suggested that the adaptive 
response to HIIT is more uniform than that observed following lower intensities of training (Bacon et al. 2013), the impact of interval training intensity on the frequency of non-responders is currently unknown. Additionally, it is not known whether an optimal intensity of HIIT exists for eliciting the greatest number of individual responders to training.

Therefore, this study had two main purposes. 1) to investigate the effect of interval intensity on training-induced adaptations in $\mathrm{VO}_{2}$ peak and $\mathrm{VO}_{2}$ kinetics and; 2) to examine the impact of interval intensity on the incidence of non-responders in $\mathrm{VO}_{2}$ peak and further characterize patterns of non-response to standardized exercise training. It was hypothesized that group and individual changes to both $\mathrm{VO}_{2}$ peak and $\mathrm{VO}_{2}$ kinetics would increase in an intensity-dependent fashion, such that submaximal HIIT elicited the smallest response and supramaximal HIIT elicited the greatest response.

\section{Materials and methods}

Experimental Design

The current study examined the impact of 3 weeks of training (4 days/week), at one of 3 different intensities, on individual and group responses in $\mathrm{VO}_{2}$ peak and $\mathrm{VO}_{2}$ kinetics. $\mathrm{VO}_{2}$ peak was determined by averaging scores obtained from $3 \mathrm{VO}_{2}$ peak tests performed during the week preceding (pre-training) and the week following training (post-training). $\mathrm{VO}_{2}$ kinetics were determined using the averaged response from 6 individual transitions to moderate-intensity exercise (80 Watts). All physiological testing and training was performed on a Monark Ergomedic 874 E stationary ergometer (Vansbro, Sweden). All experimental procedures were approved by the Health Sciences Human Research Ethics Board at Queen's University and conformed to the Declaration 
of Helsinki. Verbal and written explanation of the experimental protocol and associated risks was provided to all participants prior to obtaining written informed consent.

\section{Participants}

A total of 44 individuals were assessed for eligibility with a total of 36 completing all aspects of the study. During initial screening, individuals were only enrolled in the study if they were: between 18 and 30 years of age, non-smokers, free of cardiovascular and metabolic disease, and who self-reported completing between 60 and 150 minutes per week of structured moderate to vigorous exercise. Following screening, participants completed pre-training testing (see below for details) before being stratified based on average pre-training $\mathrm{VO}_{2}$ peak scores. Participants were subsequently randomized from within their strata into one of 3 training groups to ensure that pre-training $\mathrm{VO}_{2}$ peak did not differ between groups. Participant characteristics are presented in Table 1.

Pre- and Post-Training Testing

$\mathrm{VO}_{2}$ peak

In the week preceding training (pre), and the week following training (post), participants reported to the lab on 3 separate occasions, each separated by 24 hours, to complete a $\mathrm{VO}_{2}$ peak incremental ramp test to exhaustion. Prior to the initiation of each ramp protocol participants completed 2 transitions from loadless cycling to 80 watts (W) ( 5 minutes of loadless cycling at 80 RPM followed by a step transition to $80 \mathrm{~W}$ for 5 minutes). These transitions ( 6 in total; 2 per ramp test) were used for the determination of the $\mathrm{VO}_{2}$ kinetics response before and after training. Following the completion of the second 5-minute transition to $80 \mathrm{~W}$ the $\mathrm{VO}_{2}$ peak protocol was initiated by adding $25 \mathrm{~W}$ per minute as has been described in detail previously (Edgett et al. 2013a, 2013b). Gas 
exchange was measured throughout each test with a metabolic cart (Moxus AEI Technologies, Pittsburgh, PA), with $\mathrm{VO}_{2}$ peak calculated, as the highest value of continuous 30 second averages observed during the protocol. RPM were collected continuously throughout the test and WRpeak was calculated using the average WR from the same 30 second period as used for the determination of $\mathrm{VO}_{2}$ peak (ie. the highest continuous 30 second average of $\mathrm{VO}_{2}$ ). Pre- (and post-) training $\mathrm{VO}_{2}$ peak values presented in this manuscript are the average of the values obtained during the 3 tests completed during each week of testing.

\section{Training Protocols}

All participants completed training 4 days per week for 3 weeks. All training sessions consisted of a 1 minute loadless warm-up, multiple 1 minute intervals targeting either $80 \%$ (LO), 115\% (MID) or 150\% (HI) of pre-training WRpeak separated by 1 minute of loadless cycling, and a cool down period. Participants were instructed to maintain a cadence of 80 RPM throughout all training sessions including warm-ups, loadless rest periods between intervals, and cool downs. Training protocols were matched for total external work (kcal) with all participants completing the amount of external work estimated to be accumulated during 8 intervals at $\sim 100 \%$ of peak aerobic power in week one, 10 intervals at $\sim 100 \%$ in week 2 , and 12 intervals at $\sim 100 \%$ in week 3 . Target external work was calculated on an individual basis using the average peak aerobic power over the same 30 -second period used for determining $\mathrm{VO}_{2}$ peak, during each of the 3 pretraining $\mathrm{VO}_{2}$ peak tests. When RPM fell below 80 RPM, resulting in the target external work not being achieved in the expected number of intervals, additional intervals were added until the target amount of external work $( \pm 5 \%)$ was completed. RPM were 
collected continuously during all training sessions and was used to calculate average WR for each interval. Heart rate (HR) was recorded at the completion of each interval and total external work (kcal) was recorded following each interval to ensure target work for each training session was achieved. Rate of perceived exertion (RPE) was collected immediately following the completion of the final interval of each training session.

\section{Determination of Typical Error}

In order to determine typical error (TE) for $\mathrm{VO}_{2}$ peak, 8 recreationally active participants ( $\mathrm{n}=8$; age, $21 \pm 0.76 \mathrm{yrs}$; BMI, $21 \pm 2.25 \mathrm{~kg} / \mathrm{m}^{2}$; $\mathrm{VO}_{2}$ peak, $44 \pm 6 \mathrm{~mL} \mathrm{O}_{2} / \mathrm{kg} / \mathrm{min}$ ) reported to the lab on 2 separate occasions separated by at least a week. On each visit to the lab participants performed identical incremental ramp tests to volitional fatigue as described above and reported in detail previously (Edgett et al. 2013b, Zelt et al. 2014). $\mathrm{VO}_{2}$ peak was determined for each test and the resulting values were utilized to calculate TE.

Typical error of measurement was calculated for $\mathrm{VO}_{2}$ peak as described previously (Hopkins 2000) utilizing the following equation:

$$
T E=S D_{\text {diff }} / \sqrt{2}
$$

Where $\mathrm{SD}_{\text {diff }}$ is the variance (standard deviation) of the difference scores observed between the 2 repeated tests. A non-responder for $\mathrm{VO}_{2}$ peak was defined as an individual who failed to demonstrate an increase or decrease that was greater than $2 \mathrm{TE}( \pm 1.74$ $\mathrm{mL} / \mathrm{kg} / \mathrm{min}$ ) away from 0 . This cut-point was used as it represents a threshold past which the corresponding odds of a real change are 12 to 1 (Hopkins 2000).

$\mathrm{VO}_{2}$ kinetics 
$\mathrm{VO}_{2}$ kinetics were determined as described previously (Whipp et al. 1982, Rossiter et al. 2001). Briefly, breath-by-breath gas exchange data were collected and averaged into 10 -second bins for each of the 6 transitions to $80 \mathrm{~W}$ during pre- and posttraining testing. The data from each transition were subsequently time-aligned and the 6 transitions were ensemble averaged to yield a single profile for each subject. The ontransient response to exercise was modeled using a mono-exponential of the form:

$$
Y(t)=Y(B S L)+A M P x\left[1-e^{-(t-T D) / \tau}\right]
$$

where $\mathrm{Y}(\mathrm{t})$ represents the variable at any time $(\mathrm{t})$; $\mathrm{Y}(\mathrm{BSL})$ is the baseline value of $\mathrm{Y}$ before the step increase in WR; AMP, the amplitude (i.e., steady-state increase in Y above baseline); $\tau$, the time constant (i.e., the time taken to reach $63 \%$ of the steady-state response); and $\mathrm{TD}$ is the time delay. $\mathrm{VO}_{2}$ data were fit from the phases $1-2$ transition to the end of exercise, as previously described (Rossiter et al. 2001). $80 \mathrm{~W}$ represented a low relative intensity for all participants (see Table 3 ), and combined with visual inspection of the $\mathrm{VO}_{2}$ response and the residuals resulting from the mono-exponential curve fit (see Figure 2A for a representative response), the transition to $80 \mathrm{~W}$ appears to fall within the moderate-intensity domain for all participants at Pre and Post.

\section{Total Weekly Energy Expenditure}

During pre-training and throughout training, physical activity completed outside the lab was tracked using the Physical Activity Recall (PAR) Survey (Sallis 1997). On each occasion that participants reported to the lab they completed a PAR for the days since their last visit to the lab. This required participants to complete a PAR prior to completion of each $\mathrm{VO}_{2}$ peak test during pre-training testing and prior to each training session during the exercise intervention. Self-reported daily physical activity was 
identified as moderate, hard or very hard activities. These values were then utilized to estimate total daily and weekly energy expenditure (TWEE; $\mathrm{kcal} / \mathrm{kg} / \mathrm{wk}$ ) as described previously (Sallis 1997).

\section{Statistical Analysis}

A 2-way repeated measures ANOVA was used to compare the effect of training (time) and intensity (group) on $\mathrm{VO}_{2}$ peak, $\mathrm{VO}_{2}$ kinetics and TWEE. Significant interaction effects were deconstructed using a Bonferroni post-hoc test to examine within group changes and a one-way ANOVA to determine differences in the magnitude of change between groups. Similarities in pre-training TWEE were confirmed using a one-way ANOVA. Linear regression was used to determine the relationship between the change in $\mathrm{VO}_{2}$ peak and $\mathrm{VO}_{2}$ kinetics. All statistical analysis was performed using GraphPad Prism v 5.01 (GraphPad Software Inc., La Jolla, CA, USA). Statistical significance was accepted at $p<0.05$ and all data are presented as means \pm SEM.

\section{Results}

\section{Participant Adherence}

Attendance and completion of training sessions was 100\% in all groups for participants who completed the full study protocol. Two participants dropped out of the study following pre-training testing and 5 participants dropped out during training (LO, 2; MID, 3). Participant and training protocol characteristics are presented in Table 1 and Table 2, respectively.

Aerobic Exercise Capacity

A main effect of training $(p<0.05)$, and a significant interaction effect was observed for $\mathrm{VO}_{2}$ peak (Fig. 1A) and WRpeak (Table 1). Deconstruction of the interaction 
effect for $\mathrm{VO}_{2}$ peak revealed that the change in $\mathrm{VO}_{2}$ peak was greater $(p<0.05)$ in the MID group than the LO group; however, no differences were observed between the HI group and either the MID or LO groups $\left(\Delta \mathrm{VO}_{2}\right.$ peak; LO, $2.7 \pm 0.7 \mathrm{ml} / \mathrm{kg} / \mathrm{min}$; MID, 5.8 \pm 0.7 ; HI, $4.2 \pm 1.0$; Fig. 1B). No difference was observed for the peak HR achieved between Pre and Post for any of the training groups (LO, Pre $187 \pm 3$, Post $188 \pm 4$; MID, Pre $194 \pm 2$, Post $193 \pm 2$; HIGH, Pre $183 \pm 4$, Post $183 \pm 3$ ). The proportion of responders within each group was evaluated using our responder criteria $(2 \mathrm{x}$ Typical Error $[\mathrm{TE}=0.87 \mathrm{ml} / \mathrm{kg} / \mathrm{min} ; 2 \times \mathrm{TE}=1.74 \mathrm{ml} / \mathrm{kg} / \mathrm{min}])$. The greatest proportion of responders was observed in the MID group (Fig. 1C-E; LO, 8/12; MID, 12/13; HI, 9/11). No relationship was observed between the individual changes in peak $\mathrm{HR}$ and $\mathrm{VO}_{2}$ peak following training $\left(p=0.89, \mathrm{r}^{2}<0.001\right)$. $\mathrm{VO}_{2}$ Kinetics

A significant $(p<0.05)$ main effect of training was observed for $\tau \mathrm{VO}_{2}$ (Fig. 2B) but no interaction effect was present. Linear regression revealed no relationship $(p=0.26$; $\mathrm{r}^{2}=0.04$ ) between the training-induced increases in $\mathrm{VO}_{2}$ peak and the reduction in $\tau \mathrm{VO}_{2}$ (Fig. 2C).

\section{Total Weekly Energy Expenditure}

No differences in TWEE ( $\mathrm{kcal} / \mathrm{kg} / \mathrm{wk})$ were observed between groups at pretesting (Fig. 3A). There was no group or training effects for TWEE during the 3-week training period ( $p>0.05$; Fig. 2B). There was a significant $(p<0.05)$ group effect for moderate intensity physical activity with the MID group reporting significantly more moderate intensity physical activity during the 3 week training period than both the LO 
and HI. There were no differences between groups for the amount of light, hard or very hard physical activity reported during training.

\section{Discussion}

The current study examined the impact of three different intensities of workmatched interval training on both group and individual changes in $\mathrm{VO}_{2}$ peak and $\mathrm{VO}_{2}$ kinetics. While no difference in the increase in $\mathrm{VO}_{2}$ peak following training was observed between the MID and HI groups, only the MID group demonstrated an increase in $\mathrm{VO}_{2}$ peak that was greater than the LO group. Additionally, we observed the highest incidence of responders in the MID group (12/13). Thus, interval training at an intensity near $\mathrm{VO}_{2}$ peak (ie. MID) may represent the achievement of an adaptive threshold for improvements in aerobic capacity when external work is matched, with further increases in training intensity providing no further adaptive benefit. Interestingly, we did not observe a relationship between individual changes in $\mathrm{VO}_{2}$ peak and $\mathrm{VO}_{2}$ kinetics. These results suggest that the mechanisms underlying training-induced increases in $\mathrm{VO}_{2}$ peak and $\mathrm{VO}_{2}$ kinetics differ, and that individuals can demonstrate divergent responses in the cardiovascular adaptations induced by exercise training.

The impact of interval training intensity on $\mathrm{VO}_{2}$ peak

Increasing the intensity of submaximal exercise from $70 \%$ of maximal heart frequency $\left(\mathrm{Hf}_{\max }\right)$ to $90 \%$ of $\mathrm{Hf}_{\max }$ (Tjønna et al. 2008) and increasing interval intensity from $70 \%$ of peak WR to $100 \%$ of peak WR (Boyd et al. 2013) are both associated with greater improvements in $\mathrm{VO}_{2}$ peak. In addition, low-volume sprint interval training (SIT) 
is as effective as submaximal endurance training for inducing improvements to $\mathrm{VO}_{2}$ peak, $\mathrm{VO}_{2}$ kinetics and skeletal muscle oxidative capacity despite substantially less energy expenditure (Gibala and McGee 2008, McKay et al. 2009, McRae et al. 2012). While several investigators have examined work-matched training protocols; their results are equivocal. For example, Poole and Gaesser (1985) found no effect of training intensity on improvements in $\mathrm{VO}_{2}$ max and lactate threshold (LT) between three work-matched exercise groups, but did find that improvements in ventilatory threshold (VT) were greatest following training at $105 \%$ of $\mathrm{VO}_{2}$ max compared to each $\sim 50 \% \mathrm{VO}_{2} \max$ and $\sim 70 \%$ of $\mathrm{VO}_{2}$ max. Conversely, Tjønna and colleagues (2008) observed a greater increase in $\mathrm{VO}_{2}$ peak training at $90 \%$ than $70 \%$ of $\mathrm{Hf}_{\max }$ when training energy expenditure was matched, however, these investigators compared interval and continuous training protocols, and, to our knowledge, the current study is the first to compare work-matched supramaximal and submaximal HIIT protocols. Our observation that the MID group and not the $\mathrm{HI}$ group induced significantly greater increase in $\mathrm{VO}_{2}$ peak than the LO group, but that MID and HI did not differ, suggests that, when comparing work-matched protocols, training at intensities around $\mathrm{VO}_{2}$ peak may represent a threshold intensity above which further increases in training intensity do not provide additional adaptive benefit.

It should be noted that the current study examined the change in $\mathrm{VO}_{2}$ peak following a relatively brief ( 3 week) training intervention, and whether the trends observed in the current study would extend to longer training interventions remains an important area for future study. It is also important to note that the work rate targets utilized in the current study were derived from a $25 \mathrm{~W} / \mathrm{min}$ ramp protocol, and that 
application of these results in future studies and in the general population should take this into account. Thus, our results should be interpreted within the context of the ramp protocol utilized in this study, and applications using relative WR derived from different protocols should be made with caution.

While the underlying mechanisms that may explain this apparent plateau in training induced adaptations remain unknown, one possibility is the change in $\mathrm{pH}$ that accompanies high intensity exercise (Howlett et al. 1998). Evidence from both rats (Bishop et al. 2010) and humans (Edge et al. 2006) suggest that limiting the reduction in $\mathrm{pH}$ associated with high-intensity exercise via pretraining ingestion of sodium bicarbonate $\left(\mathrm{NaHCO}_{3}\right)$ can improve the adaptive response. Further, a recent report has demonstrated that elevated $\mathrm{pH}$ during submaximal interval exercise is associated with greater increases in peroxisome proliferator-activated-receptor $\gamma$ co-activator (PGC-1 $\alpha$ ) gene expression (Percival et al. 2015). We have recently observed a significantly greater increase in post-exercise PGC-1 $\alpha$ gene expression following near-maximal than supramaximal intervals (Edgett et al. 2013a) suggesting that the plateau in the $\mathrm{VO}_{2}$ peak response observed in the current study may result from $\mathrm{pH}$ mediated impairments in skeletal muscle adaptation. At present this hypothesis is speculative, but represents an interesting direction for future study.

Alternatively, the differences in moderate intensity physical activity (PA) observed between MID and HI may help explain why a greater increase in VO2peak was not observed following HI. Higher levels of self-reported weekly moderate and vigorous intensity exercise are associated with significantly greater changes in $\mathrm{VO}_{2}$ peak $(\mathrm{Nes}$ et al., 2012) and greater amounts of habitual light PA have also been reported to augment 
increases in $\mathrm{VO}_{2}$ peak following an endurance training intervention (Hautala et al. 2012). Given that self-report questionnaires on PA tend to overestimate PA levels (Boon et al. 2010) the observed differences in the current study should be interpreted with caution, however, they do provide preliminary evidence supporting a potential role for external PA in determining the magnitude of training induced increases in $\mathrm{VO}_{2}$ peak. Future studies should utilize objective means (i.e. accelerometers) to further investigate the relationship between habitual PA and training-induced improvements in $\mathrm{VO}_{2}$ peak.

The role of training intensity on the incidence of non-responders

Reports investigating the prevalence of non-responders to exercise training have cited differences in subject characteristics such as age (Hautala et al. 2003), sex (Parker et al. 2010), and initial fitness level (Cunningham et al. 1987) as contributors to heterogeneous responses in $\mathrm{VO}_{2}$ peak. The present study demonstrated that the incidence of responders, as characterized by a threshold increase of $2 \mathrm{TE}$ in $\mathrm{VO}_{2}$ peak $( \pm 1.74$ $\mathrm{mL} / \mathrm{kg} / \mathrm{min})$, was higher in the MID $(12 / 13 ; 92 \%)$ than the HI $(9 / 11 ; 82 \%)$ and LO $(8 / 12$; $67 \%$ ) groups. The increase in responders observed when increasing intensity from the LO to the MID condition (67-92\%) suggests that higher intensities of training may induce meaningful responses in those with low sensitivity to exercise, similar to the impact of higher volumes of submaximal exercise (Sisson et al. 2009). Importantly, while these data suggests the potential for increased rates of responders following higher intensities of interval training the sample size examined in the current work was relatively small, and much larger samples need to be examined before the relationship between interval intensity and rates of non-response can be commented on with certainty. However, the 
present results present interesting preliminary findings, and highlight this area as an important one for future study.

The lack of correlation observed between change scores of $\mathrm{VO}_{2}$ peak and $\mathrm{VO}_{2}$ kinetics observed in the present study are in agreement with observations made previously (Wilkerson et al. 2011, Edgett et al. 2013b). While it is possible that our reporting $\mathrm{VO}_{2}$ peak rather than $\mathrm{VO}_{2}$ max may have masked a relationship between maximal aerobic capacity and $\mathrm{VO}_{2}$ kinetics, this seems unlikely given previous observations that $\mathrm{VO}_{2}$ peak is likely to be a valid index of $\mathrm{VO}_{2} \mathrm{max}$ (Day et al. 2003), particularly in 'well-motivated' young, healthy, physically active participants (Poole et al. 2008) similar to those examined in the current study. The lack of correlation found in the present study reinforces the notion that these two markers of whole body oxidative metabolism during exercise can dissociate following training. This is likely due to $\mathrm{VO}_{2}$ kinetics being heavily determined by peripheral factors, namely an inertia in oxidative metabolism at the site of the muscle (Grassi 2001), and $\mathrm{VO}_{2}$ peak typically being attributed to cardiac function and oxygen delivery (Richardson et al. 1993). Previous research has provided evidence supporting individual patterns of response to each MICT (Scharhag-Rosenberger et al. 2012) and SIT (Gurd et al. 2015), whereby individuals who do not demonstrate improvements in $\mathrm{VO}_{2}$ peak are likely to improve in other variables associated with training (i.e. LT, endurance performance, submaximal exercise HR, individual anaerobic threshold). In the context of the current study, the existence of global non-responders (i.e. individuals who do not demonstrate any adaptive response following exercise training) to HIIT are unlikely. This suggestion may be clarified in future studies, which compare $\mathrm{VO}_{2}$ peak and $\mathrm{VO}_{2}$ kinetics as well as LT, VT, and gas 
exchange threshold (GET). These results are consistent with previous observations that a low response to training in $\mathrm{VO}_{2}$ peak is not associated with a low response in several other adaptations associated with metabolic control and/or exercise performance (Vollaard et al. 2009, Scharhag-Rosenberger et al. 2012). Thus, the present study provides preliminary evidence suggesting that individual patterns of response are prevalent following HIIT and highlights the need for future research to identify the other potential training outcomes necessary to adequately characterize responders and non-responders to aerobic exercise training.

\section{Conclusion}

The current study demonstrated the effect of work-matched HIIT on group and individual changes in $\mathrm{VO}_{2}$ peak and $\mathrm{VO}_{2}$ kinetics across intensities ranging from submaximal to supramaximal. Our results demonstrated no further gains in $\mathrm{VO}_{2}$ peak when increasing intensity from that performed by the MID group $(\sim 115 \%$ peak WR $)$ to that of the HI group $(\sim 150 \%$ peak WR), while the greatest incidence of responders was also found in the MID condition. These results suggest that training at intensities around $\mathrm{VO}_{2}$ peak may be optimal for work-matched HIIT. Additional results included a dissociation between individual changes in $\mathrm{VO}_{2}$ peak and $\mathrm{VO}_{2}$ kinetics, reflective of different underlying mechanisms regulating these measures of whole body oxidative metabolic adaptation, and providing preliminary support for the measurement of numerous training outcomes following training interventions. Future studies should compare outcomes such as $\mathrm{VO}_{2}$ peak, $\mathrm{VO}_{2}$ kinetics, VT, LT and mitochondrial markers of skeletal muscle adaptation in order to accurately characterize individual patterns of 
response following HIIT. Additionally, utilizing a longer training protocol than 3 weeks may allow for greater adaptation and thus further clarity regarding the impact of interval training intensity on the adaptive response to exercise.

\section{Conflict of interest}

The authors declare that there are no conflicts of interest associated with this manuscript. 


\section{References}

Bacon, A.P., Carter, R.E., Ogle, E.A., and Joyner, M.J. 2013. VO2max trainability and high intensity interval training in humans: a meta-analysis. PLoS One 8(9). doi: 10.1371/journal.pone.0073182.

Barstow, T.J., Lamarra, N., and Whipp, B.J. 1990. Modulation of muscle and pulmonary O2 uptakes by circulatory dynamics during exercise. J. Appl. Physiol. 68(3): 979989.

Berger, N.J. a, Tolfrey, K., Williams, A.G., and Jones, A.M. 2006. Influence of continuous and interval training on oxygen uptake on-kinetics. Med. Sci. Sports Exerc. 38(3): 504-512. doi: 10.1249/01.mss.0000191418.37709.81.

Bishop, D.J., Thomas, C., Moore-Morris, T., Tonkonogi, M., Sahlin, K., and Mercier, J. 2010. Sodium bicarbonate ingestion prior to training improves mitochondrial adaptations in rats. Am. J. Physiol. Endocrinol. Metab. 299(2): E225-E233. doi: 10.1152/ajpendo.00738.2009.

Boon, R.M., Hamlin, M.J., Steel, G.D., and Ross, J.J. 2010. Validation of the New Zealand Physical Activity Questionnaire (NZPAQ-LF) and the International Physical Activity Questionnaire (IPAQ-LF) with accelerometry. Br. J. Sports Med. 44(10): 741-746. doi: 10.1136/bjsm.2008.052167.

Bouchard, C., An, P., Rice, T., Skinner, J.S., Wilmore, J.H., Gagnon, J., Pérusse, L., Leon, A.S., and Rao, D.C. 1999. Familial aggregation of VO2max response to exercise training: results from the HERITAGE Family Study. J. Appl. Physiol. 87(3): 1003-1008.

Bouchard, C., and Rankinen, T. 2001. Individual differences in response to regular physical activity. Med. Sci. Sports Exerc. 33(6): S446-S451. doi: 10.1097/00005768-200106001-00013.

Boyd, J.C., Simpson, C. a., Jung, M.E., and Gurd, B.J. 2013. Reducing the intensity and volume of interval training diminishes cardiovascular adaptation but not mitochondrial biogenesis in overweight/obese men. PLoS One 8(7): e68091. doi: 10.1371/journal.pone.0068091.

Buford, T.W., Roberts, M.D., and Church, T.S. 2013. Toward exercise as personalized medicine. Sport. Med. 43(3): 157-165. doi: 10.1007/s40279-013-0018-0.

Burgomaster, K.A., Hughes, S.C., Heigenhauser, G.J.F., Bradwell, S.N., and Gibala, M.J. 2005. Six sessions of sprint interval training increases muscle oxidative potential and cycle endurance capacity in humans. J. Appl. Physiol. 98(6): 1985-1990. doi: 10.1152/japplphysiol.01095.2004.

Burke, J., Thayer, R., and Belcamino, M. 1994. Comparison of effects of two intervaltraining programmes on lactate and ventilatory thresholds. Br. J. Sports Med. 28(1): $18-21$.

Carter, H., Jones, A.M., Barstow, T.J., Burnley, M., Williams, C., and Doust, J.H. 2000. 
Effect of endurance training on oxygen uptake kinetics during treadmill running. J. Appl. Physiol. 89(5): 1744-1752.

Cocks, M., Shaw, C.S., Shepherd, S.O., Fisher, J.P., Ranasinghe, A.M., Barker, T.A., Tipton, K.D., and Wagenmakers, A.J.M. 2013. Sprint interval and endurance training are equally effective in increasing muscle microvascular density and eNOS content in sedentary males. J. Physiol. 591(3): 641-656. doi:

10.1113/jphysiol.2012.239566.

Cunningham, D.A., Rechnitzer, P.A., Howard, J.H., and P, D.A. 1987. Exercise training of men at retirement: a clinical trial. J. Gerontol. 42(1): 17-23.

Day, J.R., Rossiter, H.B., Coats, E.M., Skasick, a, and Whipp, B.J. 2003. The maximally attainable VO2 during exercise in humans: the peak vs. maximum issue. J. Appl. Physiol. 95(5): 1901-1907. doi: 10.1152/japplphysiol.00024.2003.

Eddy, D.O., Sparks, K.L., and Adelizi, D.A. 1977. The effects of continous and interval training in women and men. Eur. J. Appl. Physiol. Occup. Physiol. 92: 83-92.

Edge, J., Bishop, D., and Goodman, C. 2006. Effects of chronic NaHCO3 ingestion during interval training on changes to muscle buffer capacity, metabolism, and short-term endurance performance. J. Appl. Physiol. 101(3): 918-925. doi: 10.1152/japplphysiol.01534.2005.

Edgett, B.A., Foster, W.S., Hankinson, P.B., Simpson, C. a., Little, J.P., Graham, R.B., and Gurd, B.J. 2013a. Dissociation of increases in PGC-1 $\alpha$ and its regulators from exercise intensity and muscle activation following acute exercise. PLoS One 8(8): e71623. doi: 10.1371/journal.pone.0071623.

Edgett, B.A., Ross, J.E.D., Green, A.E., MacMillan, N.J., Milne, K.J., and Gurd, B.J. 2013b. The effects of recreational sport on VO2peak, VO2 kinetics and submaximal exercise performance in males and females. Eur. J. Appl. Physiol. 113(1): 259-266. doi: 10.1007/s00421-012-2435-4.

Gibala, M.J., and McGee, S.L. 2008. Metabolic adaptations to short-term high-intensity interval training: a little pain for a lot of gain? Exerc. Sport Sci. Rev. 36(2): 58-63. doi: 10.1097/JES.0b013e318168ec1f.

Gillen, J.B., and Gibala, M.J. 2014. Is high-intensity interval training a time-efficient exercise strategy to improve health and fitness? Appl. Physiol. Nutr. Metab. 39(3): 409-12. doi: 10.1139/apnm-2013-0187.

Grassi, B. 2001. Regulation of oxygen consumption at exercise onset: is it really controversial? Exerc. Sport Sci. Rev. 29(3): 134-138. doi: 10.1097/00003677200107000-00009.

Grassi, B., Poole, D.C., Richardson, R.S., Knight, D.R., Erickson, B.K., and Wagner, P.D. 1996. Muscle O2 uptake kinetics in humans: implications for metabolic control. J. Appl. Physiol. 80(3): 988-998.

Gurd, B.J., Giles, M.D., Bonafiglia, J.T., Raleigh, J.P., Boyd, J.C., Ma, J.K., Zelt, J.G.E., and Scribbans, T.D. 2015. Incidence of Non-Response and Individual Patterns of 
Response Following Sprint Interval Training. Appl. Physiol. Nutr. Metab. NRC Research Press. doi: 10.1139/apnm-2015-0449.

Hautala, A., Martinmaki, K., Kiviniemi, A., Kinnunen, H., Virtanen, P., Jaatinen, J., and Tulppo, M. 2012. Effects of habitual physical activity on response to endurance training. J. Sports Sci. 30(6): 563-569. doi: 10.1080/02640414.2012.658080.

Hautala, A.J., Kiviniemi, A.M., Mäkikallio, T.H., Kinnunen, H., Nissilä, S., Huikuri, H. V., and Tulppo, M.P. 2006. Individual differences in the responses to endurance and resistance training. Eur. J. Appl. Physiol. 96(5): 535-542. doi: 10.1007/s00421-0050116-2.

Hautala, A.J., Mäkikallio, T.H., Kiviniemi, A., Laukkanen, R.T., Nissilä, S., Huikuri, H. V, and Tulppo, M.P. 2003. Cardiovascular autonomic function correlates with the response to aerobic training in healthy sedentary subjects. Am. J. Physiol. - Hear. Circ. Physiol. 285(4): H1747-H1752. doi: 10.1152/ajpheart.00202.2003.

Hopkins, W.G. 2000. Measures of reliability in sports medicine and science. Sports Med. 30(1): $1-15$.

Howlett, R.A., Parolin, M.L., Dyck, D.J., Hultman, E., Jones, N.L., Heigenhauser, J.F., and Spriet, L.L. 1998. Regulation of skeletal muscle glycogen phosphorylase and $\mathrm{PDH}$ at varying exercise power outputs. Am. J. Regul. Integr. Comp. Physiol. 275: 418-425.

Krustrup, P., Hellsten, Y., and Bangsbo, J. 2004. Intense interval training enhances human skeletal muscle oxygen uptake in the initial phase of dynamic exercise at high but not at low intensities. J. Physiol. 559(1): 335-345. doi:

10.1113/jphysiol.2004.062232.

McKay, B.R., Paterson, D.H., and Kowalchuk, J.M. 2009. Effect of short-term highintensity interval training vs. continuous training on $\mathrm{O} 2$ uptake kinetics, muscle deoxygenation, and exercise performance. J. Appl. Physiol. 107(1): 128-138. doi: 10.1152/japplphysiol.90828.2008.

McRae, G., Payne, A., Zelt, J.G.E., Scribbans, T.D., Jung, M.E., Little, J.P., and Gurd, B.J. 2012. Extremely low volume, whole-body aerobic-resistance training improves aerobic fitness and muscular endurance in females. Appl. Physiol. Nutr. Metab. 37(6): 1124-1131. doi: 10.1139/h2012-093.

Mitchell, J.H., Sproule, B.J., and Chapman, C.B. 1958. The physiological meaning of the maximal oxygen intake test. J. Clin. Invest. 37(4): 538-547. doi: 10.1172/JCI103636.

Parker, B. a., Kalasky, M.J., and Proctor, D.N. 2010. Evidence for sex differences in cardiovascular aging and adaptive responses to physical activity. Eur. J. Appl. Physiol. 110(2): 235-246. doi: 10.1007/s00421-010-1506-7.

Percival, M.E., Martin, B.J., Gillen, J.B., Skelly, L.E., MacInnis, M.J., Green, A.E., Tarnopolsky, M. a., and Gibala, M.J. 2015. Sodium bicarbonate ingestion augments the increase in PGC-1 $\alpha$ mRNA expression during recovery from intense interval exercise in human skeletal muscle. J. Appl. Physiol.: jap.00048.2015. doi: 


\subsection{2/japplphysiol.00048.2015.}

Poole, D.C., and Gaesser, G. a. 1985. Response of ventilatory and lactate thresholds to continuous and interval training. J. Appl. Physiol. 58(4): 1115-1121.

Poole, D.C., and Jones, A.M. 2012. Oxygen Uptake Kinetics. Compr. Physiol. 2(April 2012): 933-996. doi: 10.1002/cphy.c100072.

Poole, D.C., Wilkerson, D.P., and Jones, A.M. 2008. Validity of criteria for establishing maximal O 2 uptake during ramp exercise tests. Eur. J. Appl. Physiol. 102(4): 403410. doi: 10.1007/s00421-007-0596-3.

Richardson, R.S., Poole, D.C., Knight, D.R., Kurdak, S.S., Hogan, M.C., Grassi, B., Johnson, E.C., Kendrick, K.F., Erickson, B.K., and Wagner, P.D. 1993. High muscle blood flow in man: is maximal O2 extraction compromised? J. Appl. Physiol. 75(4): 1911-1916.

Rossiter, H.B., Ward, S. a, Kowalchuk, J.M., Howe, F. a, Griffiths, J.R., and Whipp, B.J. 2001. Effects of prior exercise on oxygen uptake and phosphocreatine kinetics during high-intensity knee-extension exercise in humans. J. Physiol. 537(Pt 1): 291303. doi: PHY_12842 [pii].

Sallis, J. 1997. Seven-Day Physical Activity Recall. Med. Sci. Sport. Exerc. 29(6): S89S103. Available from http://journals.lww.com/acsmmsse/Fulltext/1997/06001/Seven_Day_Physical_Activity_Recall.18.aspx.

Scharhag-Rosenberger, F., Walitzek, S., Kindermann, W., and Meyer, T. 2012. Differences in adaptations to 1 year of aerobic endurance training: individual patterns of nonresponse. Scand. J. Med. Sci. Sport. 22(1): 113-118. doi: 10.1111/j.1600-0838.2010.01139.x.

Scribbans, T.D., Edgett, B. a., Vorobej, K., Mitchell, A.S., Joanisse, S.D., Matusiak, J.B.L., Parise, G., Quadrilatero, J., and Gurd, B.J. 2014. Fibre-specific responses to endurance and low volume high intensity interval training: Striking similarities in acute and chronic adaptation. PLoS One 9(6). doi: 10.1371/journal.pone.0098119.

Sisson, S.B., Katzmarzyk, P.T., Earnest, C.P., Bouchard, C., Blair, S.N., and Church, T.S. 2009. Volume of exercise and fitness nonresponse in sedentary, postmenopausal women. Med. Sci. Sports Exerc. 41(3): 539-545. doi: 10.1249/MSS.0b013e3181896c4e.

Tjønna, A.E., Lee, S.J., Rognmo, Ø., Stølen, T.O., Bye, A., Haram, P.M., Loennechen, J.P., Al-Share, Q.Y., Skogvoll, E., Slørdahl, S. a., Kemi, O.J., Najjar, S.M., and Wisløff, U. 2008. Aerobic interval training versus continuous moderate exercise as a treatment for the metabolic syndrome: A pilot study. Circulation 118(4): 346-354. doi: 10.1161/CIRCULATIONAHA.108.772822.

Vollaard, N.B.J., Constantin-Teodosiu, D., Fredriksson, K., Rooyackers, O., Jansson, E., Greenhaff, P.L., Timmons, J.A., and Sundberg, C.J. 2009. Systematic analysis of adaptations in aerobic capacity and submaximal energy metabolism provides a unique insight into determinants of human aerobic performance. J. Appl. Physiol. 106(5): 1479-1486. doi: 10.1152/japplphysiol.91453.2008. 
Whipp, B.J., Ward, S. a, Lamarra, N., Davis, J. a, and Wasserman, K. 1982. Parameters of ventilatory and gas exchange dynamics during exercise. J. Appl. Physiol. 52(6): $1506-1513$.

Wilkerson, D.P., Poole, D.C., Jones, a M., Fulford, J., Mawson, D.M., Ball, C.I., and Shore, a C. 2011. Older type 2 diabetic males do not exhibit abnormal pulmonary oxygen uptake and muscle oxygen utilization dynamics during submaximal cycling exercise. Am. J. Physiol. Regul. Integr. Comp. Physiol. 300(3): R685-R692. doi: 10.1152/ajpregu.00479.2010. 
Table 1. Participant Characteristics before (Pre) and following training (Post)

\begin{tabular}{|c|c|c|c|c|c|c|}
\hline & \multicolumn{2}{|l|}{ LO } & \multicolumn{2}{|l|}{ MID } & \multicolumn{2}{|l|}{ HI } \\
\hline & Pre & Post & Pre & Post & Pre & Post \\
\hline $\mathbf{N}$ & 12 & & 13 & & 11 & \\
\hline Age (yrs) & $20 \pm 1$ & & $20 \pm 1$ & & $20 \pm 1$ & \\
\hline Height (cm) & $\begin{array}{l}170 \pm \\
10\end{array}$ & & $\begin{array}{l}173 \pm \\
13\end{array}$ & & $176 \pm 9$ & \\
\hline Body mass (kg) & $67 \pm 12$ & $67 \pm 12$ & $70 \pm 14$ & $70 \pm 14$ & $75 \pm 14$ & $75 \pm 14$ \\
\hline BMI $\left(\mathrm{kg} / \mathrm{m}^{2}\right)$ & $23 \pm 2$ & $23 \pm 2$ & $23 \pm 3$ & $23 \pm 3$ & $24 \pm 3$ & $24 \pm 3$ \\
\hline $\begin{array}{l}\text { Peak aerobic power }(\mathrm{W}) \\
* \dagger\end{array}$ & $\begin{array}{l}225 \pm \\
66\end{array}$ & $\begin{array}{l}241 \pm \\
66\end{array}$ & $\begin{array}{l}228 \pm \\
61\end{array}$ & $\begin{array}{l}275 \pm \\
83\end{array}$ & $\begin{array}{l}243 \pm \\
61\end{array}$ & $\begin{array}{l}270 \pm \\
64\end{array}$ \\
\hline
\end{tabular}

Values are means \pm SEM. yrs, years; cm, centimeters; kg, kilograms; BMI, body mass index; m, meters; W, watts.

* Main effect of training $(p<0.05)$

$\uparrow$ Significant $(p<0.05)$ group $\mathrm{x}$ time interaction effect 
Table 2. Interval training protocol characteristics

\begin{tabular}{llllll}
\hline & LO & & MID & & HI \\
\cline { 2 - 2 } \cline { 5 - 6 } Intervals Performed & $11.7 \pm 0.3$ & & $8.8 \pm 0.3$ & & $7.7 \pm 0.4$ \\
Mean Interval WR (watts) & $181 \pm 3$ & & $269 \pm 7$ & & $327 \pm 32$ \\
Mean Interval Intensity (\% of peak) & $83 \pm 13$ & & $112 \pm 9$ & & $130 \pm 20$ \\
External Work (kJ) & $149 \pm 10$ & & $167 \pm 12$ & & $169 \pm 14$ \\
Average Interval HR (\% HR max) & $76 \pm 3$ & & $87 \pm 6$ & & $90 \pm 3$ \\
Rate of Perceived Exertion & $13 \pm 2$ & & $17 \pm 2$ & & $19 \pm 2$ \\
\hline
\end{tabular}

Values presented as mean \pm SEM. WR, work rate; kJ, kilojoules; HR, heart rate. 
Table 3. Summary of parameter estimates for moderate-intensity VO2 kinetics before (Pre) and after (Post) training.

\begin{tabular}{|c|c|c|c|c|c|c|}
\hline & \multicolumn{2}{|l|}{ LO } & \multicolumn{2}{|l|}{ MID } & \multicolumn{2}{|l|}{ HI } \\
\hline & Pre & Post & Pre & Post & Pre & Post \\
\hline Bsl (mL/min) & $796 \pm 56$ & $781 \pm 57$ & $835 \pm 52$ & $791 \pm 41$ & $837 \pm 69$ & $804 \pm 56$ \\
\hline $\operatorname{Amp}(\mathrm{mL} / \mathrm{min})$ & $423 \pm 31$ & $450 \pm 26$ & $434 \pm 27$ & $473 \pm 12$ & $460 \pm 21$ & $450 \pm 23$ \\
\hline $\begin{array}{l}\text { End Ex } \\
(\mathbf{m L} / \mathbf{m i n})\end{array}$ & $1219 \pm 70$ & $1231 \pm 65$ & $1269 \pm 41$ & $1264 \pm 43$ & $1297 \pm 77$ & $1255 \pm 65$ \\
\hline$\tau \mathrm{VO2}(\mathrm{sec}) *$ & $31 \pm 2$ & $26 \pm 4$ & $33 \pm 4$ & $26 \pm 4$ & $32 \pm 3$ & $25 \pm 3$ \\
\hline $\begin{array}{l}\text { Moderate WR } \\
\text { (\% VO } \mathrm{VO}_{2} \text { peak) }\end{array}$ & $39 \pm 10$ & $35 \pm 8$ & $36 \pm 11$ & $32 \pm 9$ & $34 \pm 7$ & $30 \pm 6$ \\
\hline
\end{tabular}

Values are means \pm SEM. Bsl, baseline; Amp, amplitude; Ex, exercise; WR, work rate.

* Significant $(\mathrm{P}<0.05)$ main effect of training; 


\section{Figure Legends}

Figure 1: $\mathrm{VO}_{2}$ peak $(\mathrm{mL} / \mathrm{kg} / \mathrm{min})$ values from pre-testing to post-testing are shown (A) for all three intensity groups ( $\mathrm{LO}, \mathrm{MID}$ and $\mathrm{HI}$ ). The average change in $\mathrm{VO}_{2}$ peak $(\mathrm{mL} / \mathrm{kg} / \mathrm{min})$ values from pre-testing to post-testing are also shown (B) as are the individual responses for all participants (C-E) for all three intensity groups (LO, MID and HI).

$\alpha$ Significant $(\mathrm{p}<0.05)$ main effect of training

$\beta$ Significant $(p<0.05)$ group $x$ time (i.e. training) interaction effect

* Significant $(\mathrm{p}<0.05)$ difference from baseline within group

$\uparrow$ Significant $(\mathrm{p}<0.05)$ difference between LO and MID intensity groups.

Figure 2: Absolute $\mathrm{VO}_{2}$ response with line of best fit and residuals for Pre (black circles and lines of best fit/residuals) and Post (grey circles and line of best fit/residuals) of a representative subject $(\tau$ Pre $=38 \mathrm{sec} ; \tau$ Post $=22 \mathrm{sec})$ ish shown $(\mathrm{A}) . \tau \mathrm{VO} 2(\mathrm{~s})$ values from pre-testing to post-testing (A) for all three intensity groups (LO, MID, and HI) and the relationship between the change in $\mathrm{VO}_{2}$ peak and the change in $\tau \mathrm{VO}_{2}(\mathrm{C})$ are also shown with data points above 0 reflecting an increase in $\mathrm{VO}_{2}$ peak and a faster $\mathrm{VO}_{2}$ response.

$\alpha$ Significant $(\mathrm{p}<0.05)$ main effect of training

Figure 3: Total weekly energy expenditure (TWEE) (kcal/kg/wk) reported for all three intensity groups (LO, MID and HI) during pre-testing (A) and during the three training weeks (B). The total reported moderate-intensity physical activity (PA) (kcal/kg/wk) performed during the three training weeks is also shown (C) for all three intensity groups. *Significant $(p<0.05)$ group effect such that moderate intensity PA was greater in the MID group for all 3 weeks of training. 


\section{Figure 1}
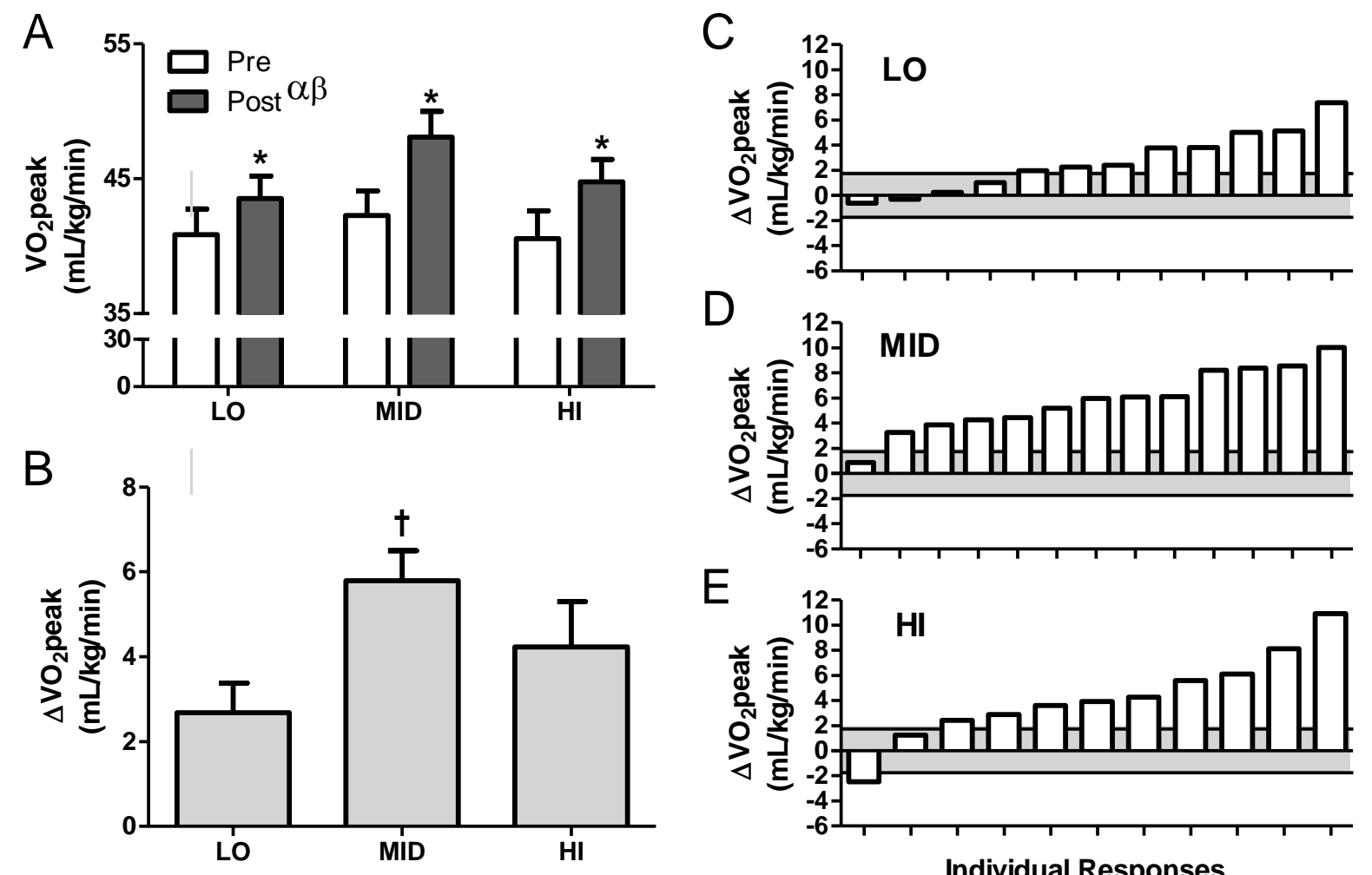

E

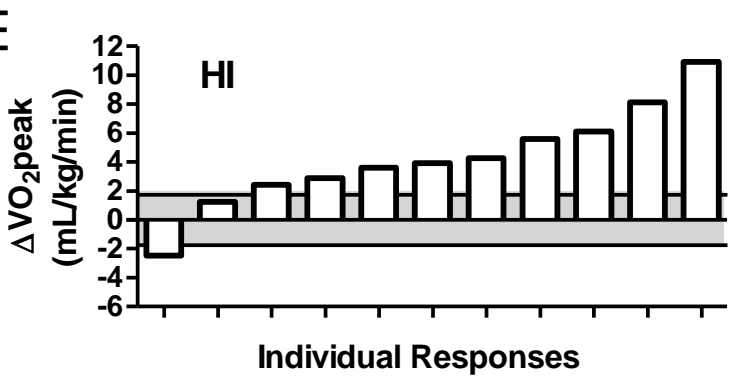




\section{Figure 2}

A
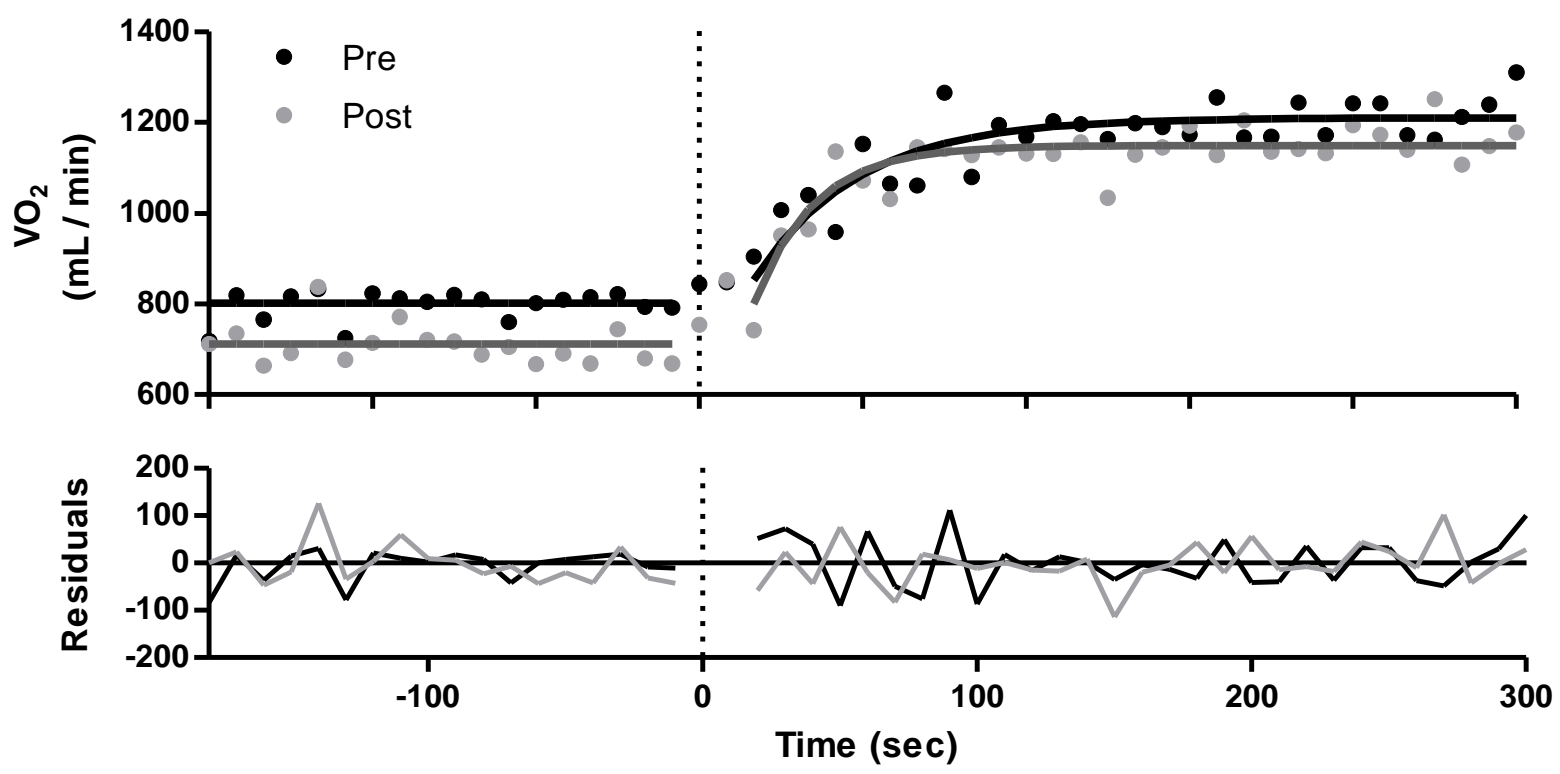

B

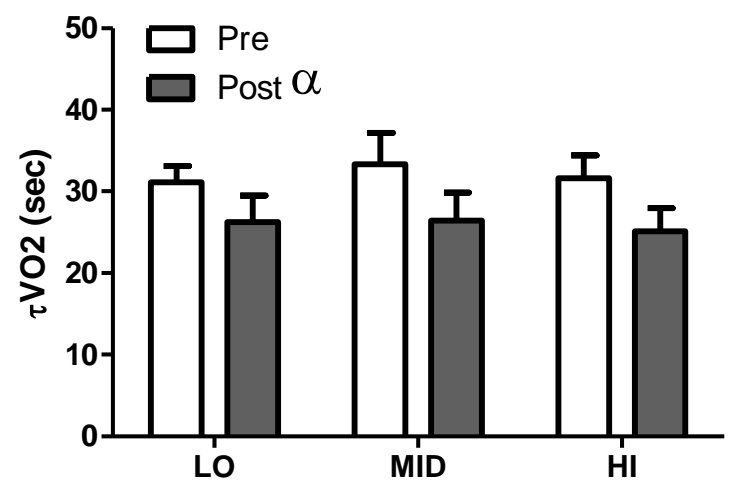

C

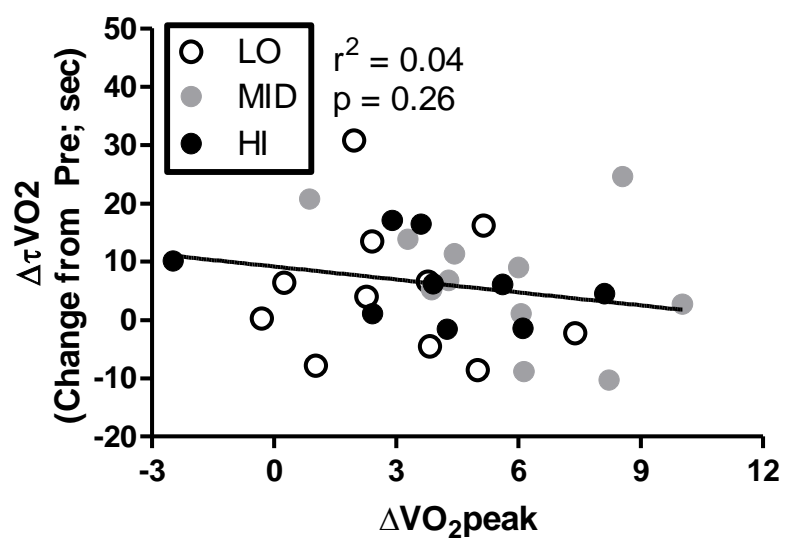

(Change from Pre; $\mathrm{mL} / \mathrm{kg} / \mathrm{min}$ ) 


\section{Figure 3}
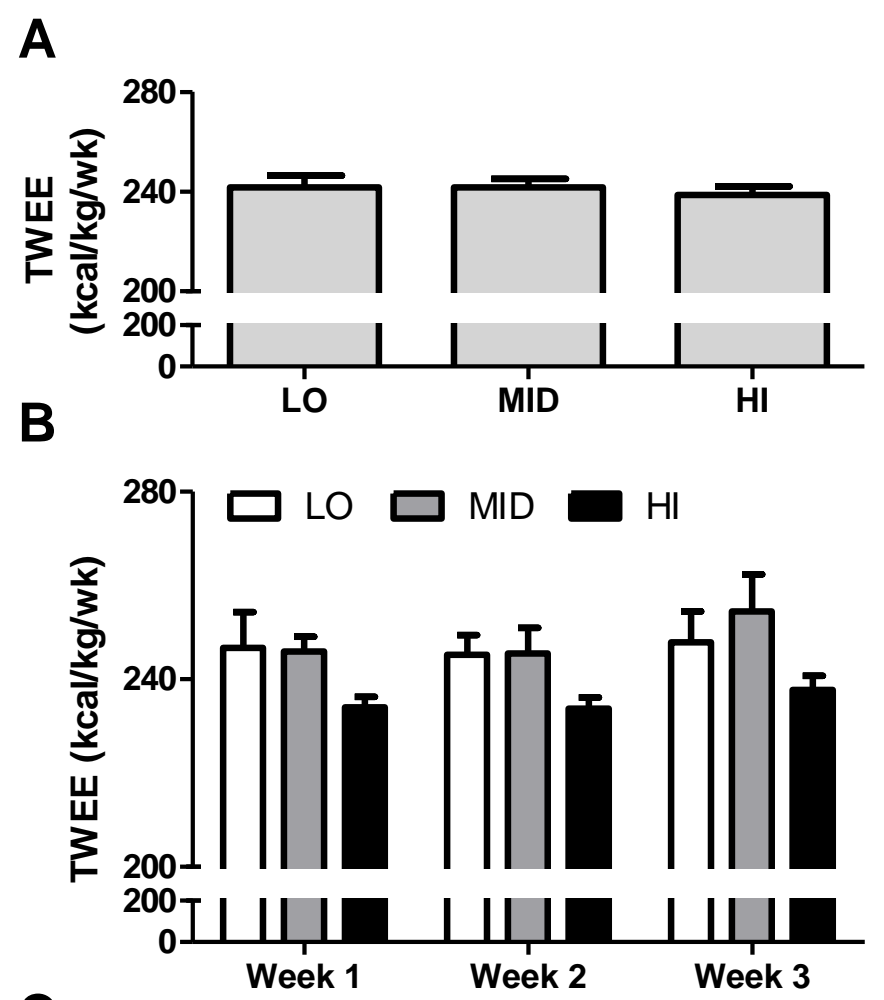

C

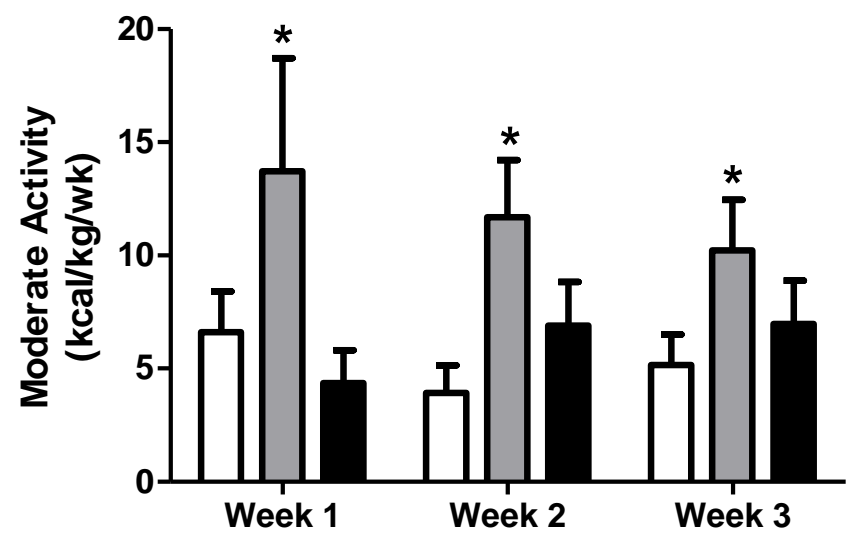

